\title{
An institutional series and contemporary review of bacterial spinal epidural abscess: current status and future directions
}

\author{
Faris Shweikeh, M.D., ${ }^{1}$ Kashif Saeed, M.D.,${ }^{2}$ Laura Bukavina, M.D.,${ }^{3}$ \\ Stephanie Zyck, M.D., ${ }^{1}$ Doniel Drazin, M.D., ${ }^{4}$ and Michael P. Steinmetz, M.D. ${ }^{5,6}$ \\ ${ }^{1}$ College of Medicine, Northeast Ohio Medical University, Rootstown, Ohio; ${ }^{2}$ Department of Physical \\ Medicine and Rehabilitation, Rush University Medical Center, Chicago, Illinois, ${ }^{3}$ Department of Medicine, \\ Summa Health System, Akron, Ohio; ${ }^{4}$ Department of Neurosurgery, Cedars-Sinai Medical Center, Los \\ Angeles, California; ${ }^{5}$ Department of Neurosciences, MetroHealth Medical Center, Cleveland, Ohio; and \\ ${ }^{6}$ Department of Neurological Surgery, Case Western Reserve University School of Medicine, Cleveland, Ohio
}

\begin{abstract}
Object. Over the past decade, the incidence of bacterial spinal epidural abscess (SEA) has been increasing. In recent years, studies on this condition have been rampant in the literature. The authors present an 11-year institutional experience with SEA patients. Additionally, through an analysis of the contemporary literature, they provide an update on the challenging and controversial nature of this increasingly encountered condition.

Methods. An electronic medical record database was used to retrospectively analyze patients admitted with SEA from January 2001 through February 2012. Presenting symptoms, concurrent conditions, microorganisms, diagnostic modalities, treatments, and outcomes were examined. For the literature search, PubMed was used as the search engine. Studies published from January 1, 2000, through December 31, 2013, were critically reviewed. Data from articles on methodology, demographics, treatments, and outcomes were recorded.

Results. A total of 106 patients with bacterial SEA were identified. The mean \pm SD age of patients was $63.3 \pm$ 13.7 years, and $65.1 \%$ of patients were male. Common presenting signs and symptoms were back pain $(47.1 \%)$ and focal neurological deficits $(47.1 \%)$. Over $75 \%$ of SEAs were in the thoracolumbar spine, and over 50\% were ventral. Approximately $34 \%$ had an infectious origin. Concurrent conditions included diabetes mellitus (35.8\%), vascular conditions (31.3\%), and renal insufficiency/dialysis (30.2\%). The most commonly isolated organism was Staphylococcus aureus (70.7\%), followed by Streptococcus spp. (6.6\%). Surgery along with antibiotics was the treatment for $63(59.4 \%)$ patients. Surgery involved spinal fusion for $19(30.2 \%)$, discectomy for $14(22.2 \%)$, and corpectomy for $9(14.3 \%)$. Outcomes were reported objectively; at a mean \pm SD follow-up time of $8.4 \pm 26$ weeks (range 0-192 weeks), outcome was good for $60.7 \%$ of patients and poor for $39.3 \%$. The literature search yielded 40 articles, and the authors discuss the result of these studies.

Conclusions. Bacterial SEA is an ominous condition that calls for early recognition. Neurological status at the time of presentation is a key factor in decision making and patient outcome. In recent years, surgical treatment has been advocated for patients with neurological deficits and failed response to medical therapy. Surgery should be performed immediately and before 36-72 hours from onset of neurological sequelae. However, the decision between medical or surgical intervention entails individual patient considerations including age, concurrent conditions, and objective findings. An evidence-based algorithm for diagnosis and treatment is suggested. (http://thejns.org/doi/abs/10.3171/2014.6.FOCUS14146)
\end{abstract}

\section{Key Words - $\quad$ spinal epidural abscess - CNS infection • spine surgery • treatment - management algorithm}

\begin{abstract}
A SPINAL epidural abscess (SEA) is a suppurative infection localized to the extradural space..$^{11,24}$ Although SEAs are quite rare, their incidence has been rising gradually over the recent decade,,${ }^{10,11,19,24,33,34}$ possibly associated with increasing intravenous drug abuse, expanding use of spinal instrumentation, and an aging population with multiple concurrent conditions. ${ }^{11,19,24}$ Clinical presentation is typically characterized by back or neck pain, fe-
\end{abstract}

\footnotetext{
Abbreviations used in this paper: ASIA = American Spinal Injury Association; MRSA = methicillin-resistant Staphylococcus aureus; $\mathrm{SEA}=$ spinal epidural abscess .
}

ver, and focal neurological deficits; however, recognition and diagnosis of SEAs can often be arduous. ${ }^{19,21,24}$ SEAs can be either primary or secondary. Primary SEAs result from hematogenous spread of pathogens from a distant focus into the epidural space; secondary SEAs occur after spinal trauma, injections, surgery, or direct inoculation of a pathogen into the epidural space..$^{46}$ The pathophysiology of primary and secondary SEAs might be closely associated with comorbidities.

In the past, treatment has consisted of urgent surgical decompression and abscess drainage along with multiple weeks of intravenous antibiotic therapy. ${ }^{0,19,23,33}$ Decompression surgery has involved laminectomy, disc- 
ectomy, fusion, and corpectomy.,15,19 Decompression also serves as a way to identify the causative microbe so that appropriate antibiotic therapy can be expeditiously administered..$^{4,15,37}$ In select patients with mild back pain and without neurological deficits, antibiotic treatment and immobilization alone can be sufficient therapy; $4,11,36,37$ these patients, however, need close monitoring because immediate operative intervention can become necessary in cases of subsequent neurological deterioration. ${ }^{4,23,32}$

Bacterial SEAs have been characterized in several case reports and case series; their epidemiology, predisposing conditions, diagnostic modalities, and approaches to management have all been described. We analyzed such patient data over an 11-year period and interconnected the results with those in the literature. Our review of the contemporary literature provides an update and sheds light on a condition that has been of great interest and challenge to neurosurgeons.

\section{Methods}

\section{Retrospective Study}

After receiving institutional review board approval, we searched the electronic medical record database at Summa Health System for all patients with a discharge diagnosis of SEA from January 2001 through February 2012. The search identified 160 patients. We excluded 54 patients: 46 patients had been readmitted for the original SEA (Fig. 1), and 8 lacked pertinent information or had been transferred to an alternate facility. For the 106 patients included in the study, each chart was reviewed from admission to discharge and assessed for presenting symptoms, predisposing factors, infecting microorganism, mode of diagnosis, mode of treatment, and outcome.

\section{Literature Review}

A literature search was conducted through the National Library of Medicine search engine, PubMed. Our aim was to find large retrospective or prospective studies of patients with bacterial SEA. Because we intended to obtain recent studies, particularly those published after the last meta-analysis published in $2000,{ }^{34}$ we included only studies that had been published from 2000 on. We excluded case reports and review articles. We included only articles published in the English language and in peer-reviewed journals. Findings from our study were compared with those from studies reported in the literature.

We began the query with the search term "spinal epidural abscess" limited to publications from January 1, 2000, through December 31, 2013. The initial search produced 1264 results. After limiting the search to publications in the English language (1087 articles), abstracts of these articles were reviewed by 3 authors (F.S., K.S., and S.Z.). This review yielded articles for detailed review and inclusion in the analysis. After examining the reference lists from the selected articles for other relevant articles, we included a total of 40 studies in the updated review. The remaining articles were not centered on SEA, discussed a different disease entity, were case reports, or were literature reviews. Data from the chosen articles on research methodology, patient age, demographics, treatments offered, and outcomes at mean follow-up time were recorded.

\section{Results}

\section{Retrospective Study}

Of the 106 patients evaluated, most (69 [65.1\%]) were male, and the mean \pm SD age of patients was $63.3 \pm 13.7$ years (range 33-89 years). The most frequent presenting symptoms were back pain in $48(45.3 \%)$ and focal neurological findings in $48(47.1 \%)$. Of the patients who had focal neurological findings at initial presentation, 25 (24.5\%) complained of paralysis and paresis of the extremities and $23(22.5 \%)$ complained of paresthesias and pain in the extremities. Other presenting signs or symptoms were fever/chills in 34 (33\%), urinary or bowel incontinence in $18(17.6 \%)$, altered mental status or lethargy in $13(12.7 \%)$, neck pain in $8(7.8 \%)$, and nausea and/or vomiting in $5(4.9 \%)$. These findings are summarized in Table 1.

We designated SEA as primary if the patient had no history of spine surgery, injections, or any other invasive spinal procedure. In our study, the cause of SEA was primary for $85(80.2 \%)$ patients and secondary for $21(19.8 \%)$. Table 2 summarizes results regarding abscess locations. Among primary SEAs, 40 (47.1\%) were lumbar, $29(34.1 \%)$ were thoracic, $15(17.6 \%)$ were cervical, and 1 $(1.2 \%)$ was sacral. Among secondary SEAs, 15 (71.4\%) were lumbar, $4(19.0 \%)$ were thoracic, $1(4.7 \%)$ was sacral, and $1(4.7 \%)$ was cervical. Of clinical relevance, 30 (37.5\%) of SEAs were dorsal and 47 (58.8\%) were ventral. Primary SEAs were slightly more likely than secondary SEAs to involve more levels (2.05 vs 1.81 , respectively).

For $36(34.0 \%)$ of the 106 patients, an infectious ori-

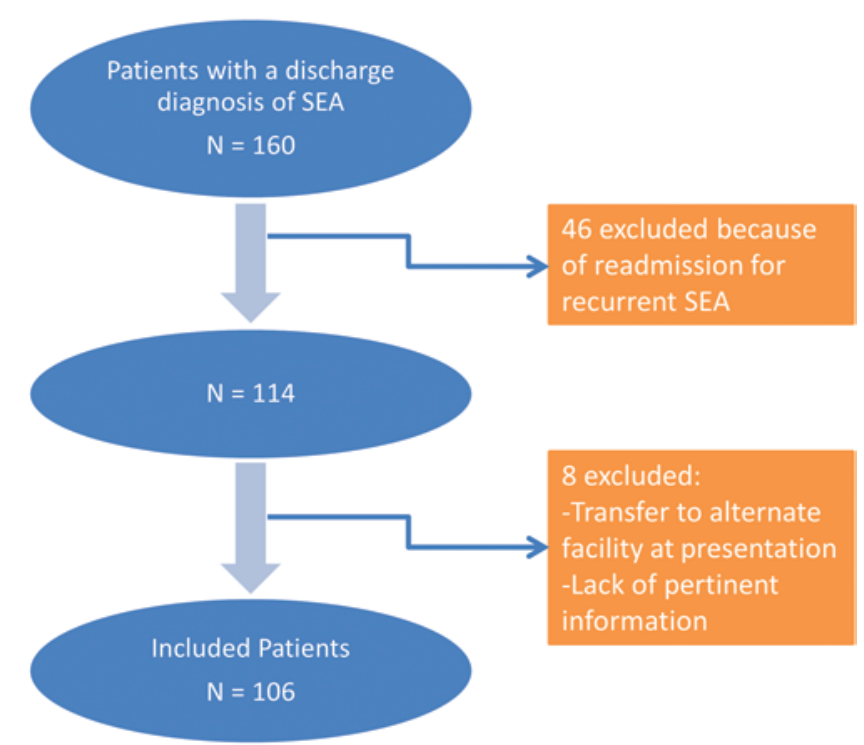

Fig. 1. Flow chart showing inclusion and exclusion criteria for the original 160 patients with a discharge diagnosis of SEA on the institutional electronic medical record. 
gin or source of the SEA was identifiable, such as infectious skin lesions, a genitourinary infectious process, a gastrointestinal focus, and oral lesions (Table 3). Because articles in the literature reported that comorbid and underlying conditions are significant factors associated with patient outcome, we assessed such factors in our patient population (Table 4). In concordance with reports in the literature, the most common comorbid condition was diabetes mellitus, found in $38(35.8 \%)$ patients in our study. The next most common conditions were vascular disease (33 [31.1\%]) patients, underlying renal insufficiency or receipt of dialysis (32 [30.2\%]), sepsis and/or bacteremia (23 [21.7\%]), and drug abuse (23 [21.7\%]). Also seen in our patient population, but with lesser frequency, were underlying malignancy, receipt of immunosuppressive therapy, chronic inflammatory diseases, and endocarditis.

For each of the 106 patients, the causative organism was determined (Fig. 2). The most common causative organism was methicillin-sensitive Staphylococcus aureus $(39.60 \%)$, followed by methicillin-resistant $S$. aureus (MRSA) (31.10\%), Streptococcus spp. (6.60\%), and coagulase-negative Staphylococcus spp. (3.80\%). Also found in some patients were Klebsiella spp. (2.80\%), Candida spp. (1.90\%), Escherichia coli (1.90\%), Aspergillus spp. (1.90\%), and Enterobacter spp. (0.94\%). Cultures were negative for $6(5.6 \%)$ patients. In terms of imaging modalities used, MRI was used most frequently (81 patients [76.4\%]), followed by CT-guided biopsy (15 [14.2\%]), and CT (7 [6.6\%]). Surgical exploration was also used for diagnosis in $3(2.8 \%)$ patients.

With regard to treatments offered and chosen surgical procedures (Table 5), $63(59.4 \%)$ patients were offered a combination of antibiotics and surgery. Of these patients, $2(3.2 \%)$ died and $61(96.8 \%)$ were ultimately discharged. Of the $33(31.1 \%)$ patients offered antibiotics alone, $4(12.1 \%)$ died and $29(87.9 \%)$ were discharged. All surgical patients received drainage, decompression, and debridement, and some also underwent fusion, corpectomy, and/or discectomy. As outlined in Table 5, 19 (30.2\%) patients underwent spinal fusion; 14 (22.2\%) un-

TABLE 1: Frequency of common presenting signs and symptoms among 106 patients with SEA

\begin{tabular}{lc}
\hline \multicolumn{1}{c}{ Sign or Symptom } & Frequency, No. $(\%)^{*}$ \\
\hline back pain & $48(47.1)$ \\
neck pain & $8(7.8)$ \\
acute & $25(24.5)$ \\
progressively worsening & $23(22.5)$ \\
fevers/chills & $34(33)$ \\
altered mental status/lethargy & $13(12.7)$ \\
focal neurological findings & $48(47.1)$ \\
paralysis/paresis in extremities & $25(24.5)$ \\
paresthesia/pain in extremities & $23(22.5)$ \\
nausea/vomiting & $5(4.9)$ \\
urinary/bowel symptoms & $18(17.6)$ \\
\hline
\end{tabular}

* Some patients presented with more than 1 sign or symptom. Percentages calculated out of 102 patients with complete data.
TABLE 2: Location of SEA with regard to primary or secondary causes in 106 patients

\begin{tabular}{cc}
\hline Location & Frequency, No. (\%) \\
\hline primary & $15(17.6)$ \\
cervical & $29(34.1)$ \\
thoracic & $40(47.1)$ \\
lumbar & $1(1.2)$ \\
sacral & $85(80.2)$ \\
total & \\
secondary & $1(4.7)$ \\
cervical & $4(19.0)$ \\
thoracic & $15(71.4)$ \\
lumbar & $1(4.7)$ \\
sacral & $21(19.8)$ \\
total & $47(58.8)$ \\
ventral & $30(37.5)$ \\
dorsal $^{*}$ & $3(3.8)$ \\
lateral/circumferential $^{*}$
\end{tabular}

* Ventral or dorsal location data were missing for 26 patients; percentages are based on 80 patients.

derwent discectomy; 9 (14.3\%) underwent corpectomy; and $46(73.0 \%)$ underwent decompressive laminectomy (with drainage and/or debridement) only.

To tangibly describe outcomes with regard to neurological function, we reported outcomes objectively, based on a previously reported objective score (Table 6). ${ }^{25} \mathrm{~Pa}$ tients were placed accordingly into 1 of 5 categories at time of most recent follow-up examination: full recovery of neurological function (29 patients), able to ambulate independently and without sphincter disturbance (8 patients), able to ambulate with assistance only and possible associated sphincter disturbance (9 patients), paraplegic or minimal recovery but bedridden (10 patients), or expired (6 patients). These outcomes were recorded at mean \pm SD follow-up time of $8.4 \pm 26$ weeks (range 0-192 weeks); most were obtained at discharge. The other patients were lost to follow-up or discharged to another facility; thus, their records could not be obtained and reported.

\section{Literature Review}

Most studies reviewed were institutional case series (Table 7). Almost all were retrospective studies.

Epidemiology. The incidence of SEA has been inTABLE 3: Identified infectious origin of SEA in 36 patients among a series of 106 patients

\begin{tabular}{lcc}
\hline \multirow{2}{*}{ Origin } & \multicolumn{2}{c}{ SEA No. } \\
\cline { 2 - 3 } & Primary & Secondary \\
\hline skin lesion & 9 & 5 \\
oral lesion/focus & 3 & 1 \\
gastrointestinal focus & 5 & 1 \\
genitourinary focus & 9 & 3 \\
\hline
\end{tabular}


TABLE 4: Comorbid and underlying conditions in 106 SEA patients

\begin{tabular}{lc}
\hline \multicolumn{1}{c}{ Concurrent Condition } & Frequency, No. $(\%)^{*}$ \\
\hline diabetes mellitus & $38(35.8)$ \\
vascular disease & $33(31.1)$ \\
renal insufficiency \&/or dialysis & $32(30.2)$ \\
sepsis \&/or bacteremia & $23(21.7)$ \\
substance abuse (alcohol, tobacco, intravenous & $23(21.7)$ \\
$\quad$ drug abuse) & \\
previous spine op & $21(19.8)$ \\
malignancy & $14(13.2)$ \\
immunosuppressive therapy \&/or chronic inflam- & $11(10.3)$ \\
$\quad$ matory disease & \\
endocarditis & $2(1.9)$ \\
\hline
\end{tabular}

* Because some patients had more than 1 concurrent condition, percentages do not add up to 100 .

creasing. Recent estimates are around $2-3$ cases per 10,000 hospital admissions. ${ }^{1,11,37,46}$ Spinal epidural abscesses can be classified into primary versus secondary. Secondary SEAs occur in patients with a history of invasive spinal procedures. ${ }^{46}$ Some have defined secondary SEA as being associated with vertebral osteomyelitis. ${ }^{20}$ Most patients are in the 50- to 70-year age range; men are more likely to be afflicted..$^{23}$ Origins or foci of infection have been identified and include pathogens in skin, oral mucosa, genitourinary system, and bowel. ${ }^{46}$ Frequently reported concurrent conditions are diabetes mellitus, previous spine surgery, intravenous drug abuse, epidural anesthesia, alcoholism, renal failure, hemodialysis, systemic chronic disorders, and immunosuppression. $3,17,21,3,43$ Factors that can be associated with a worse prognosis are older age, diabetes mellitus, neurological and mental deterioration, and dorsal location of the SEA. 1,8,9,19,23,32 Additionally, higher reoperation and mortality rates are associated with renal failure and hemodialysis. ${ }^{44,45}$ In terms

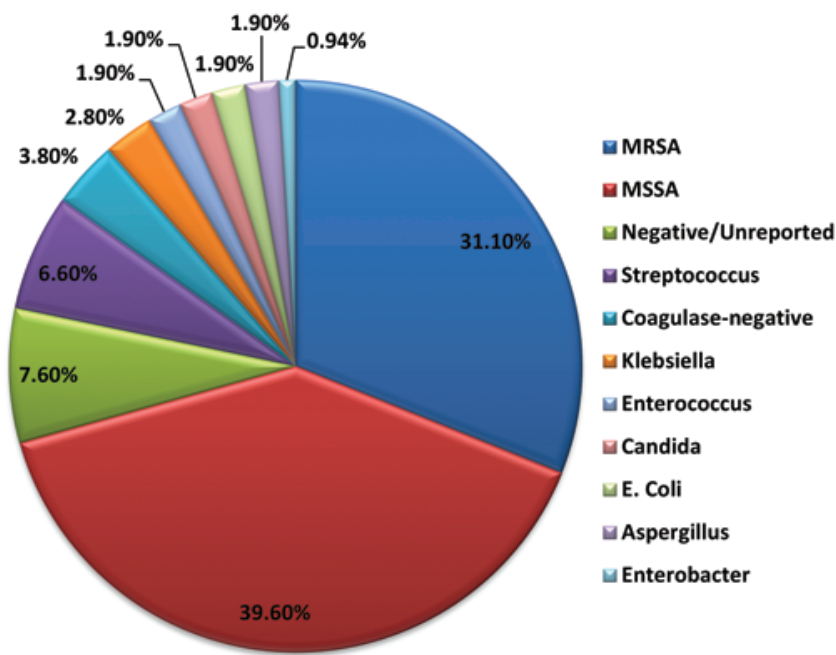

FIG. 2. Percentage of microorganisms cultured among 106 patients with SEA. Coagulase-negative = coagulase-negative Staphylococcus; E. coli $=$ Escherichia coli; MSSA = methicillin-sensitive $S$. aureus.
TABLE 5: Treatments and surgical procedures performed in SEA patients

\begin{tabular}{lc}
\hline \multicolumn{1}{c}{ Treatment } & Frequency, No. (\%) \\
\hline antibiotics alone & $33(31.1)$ \\
antibiotics + op & $63(59.4)$ \\
decompressive laminectomy only & $46(73.0)$ \\
fusion & $19(30.2)$ \\
discectomy & $14(22.2)$ \\
corpectomy & $9(14.3)$ \\
\hline
\end{tabular}

* Because some patients received a combination of fusion, corpectomy, and/or discectomy, percentages do not add up to 100 .

of pathogenic microorganisms, overwhelmingly the most common is $S$. aureus, followed by Streptococcus spp. and gram-negative bacteria. . $^{4,16,17,30,38}$ Mortality rates are 5\%$23 \% ; ;^{1,9,11,30}$ morbidity rates are $33 \%-47 \%$. 910

Presentation and Diagnosis. The most common signs and symptoms are neck and back pain, fever, and neurological deficits., ${ }^{428}$ Others, such as altered mental status and incontinence, can also be present. ${ }^{4,28}$ However, presentation is variable and can be ambiguous, leading to delayed diagnosis., ${ }^{72,14,18,27,37,41}$ Reportedly, SEA usually occurs in the thoracolumbar spine, dorsally. ${ }^{19,30,31}$ In one study, systemic symptoms were more common among those with ventral lesions, and focal deficits were more common among those with dorsal lesions. ${ }^{19}$ In the absence of contraindications, the standard imaging modality is MRI; CT myelography is an equivalent option..$^{10,33,41}$ Ultrasonography has been used intraoperatively to ensure complete abscess clearance. ${ }^{42}$ Along with increased white blood cell count, inflammatory markers such as C-reactive protein and erythrocyte sedimentation rate are usually also increased; both markers can be used to monitor treatment success $s^{10,12,18,43}$ and have also been found to predict treatment success and outcomes. . $^{32,38}$

Treatment and Outcome. Generally, antibiotics should be initiated as soon as SEA diagnosis is suspected. For $25 \%$ of patients, antibiotic therapy for less than 4 weeks is associated with treatment failure., ${ }^{4,38}$ Therapy should be culture specific, and peripheral blood cultures can be possibly used as indicators of the pathogen. ${ }^{10,17,32}$ Computed to-

TABLE 6: Outcomes after treatment for 106 SEA patients

\begin{tabular}{lc}
\hline \multicolumn{1}{c}{ Outcome } & Frequency, No. \\
\hline full recovery; neurologically intact & 29 \\
independent ambulation; no sphincter disturbance & 8 \\
ambulation w/ assistance; possible sphincter & 9 \\
$\quad$ disturbance & \\
paraplegic or minimal recovery & 10 \\
died & 6 \\
lost to follow-up* & 44 \\
\hline
\end{tabular}

* Includes patients who were discharged to an extended nursing care facility. 


\section{Update on spinal epidural abscess}

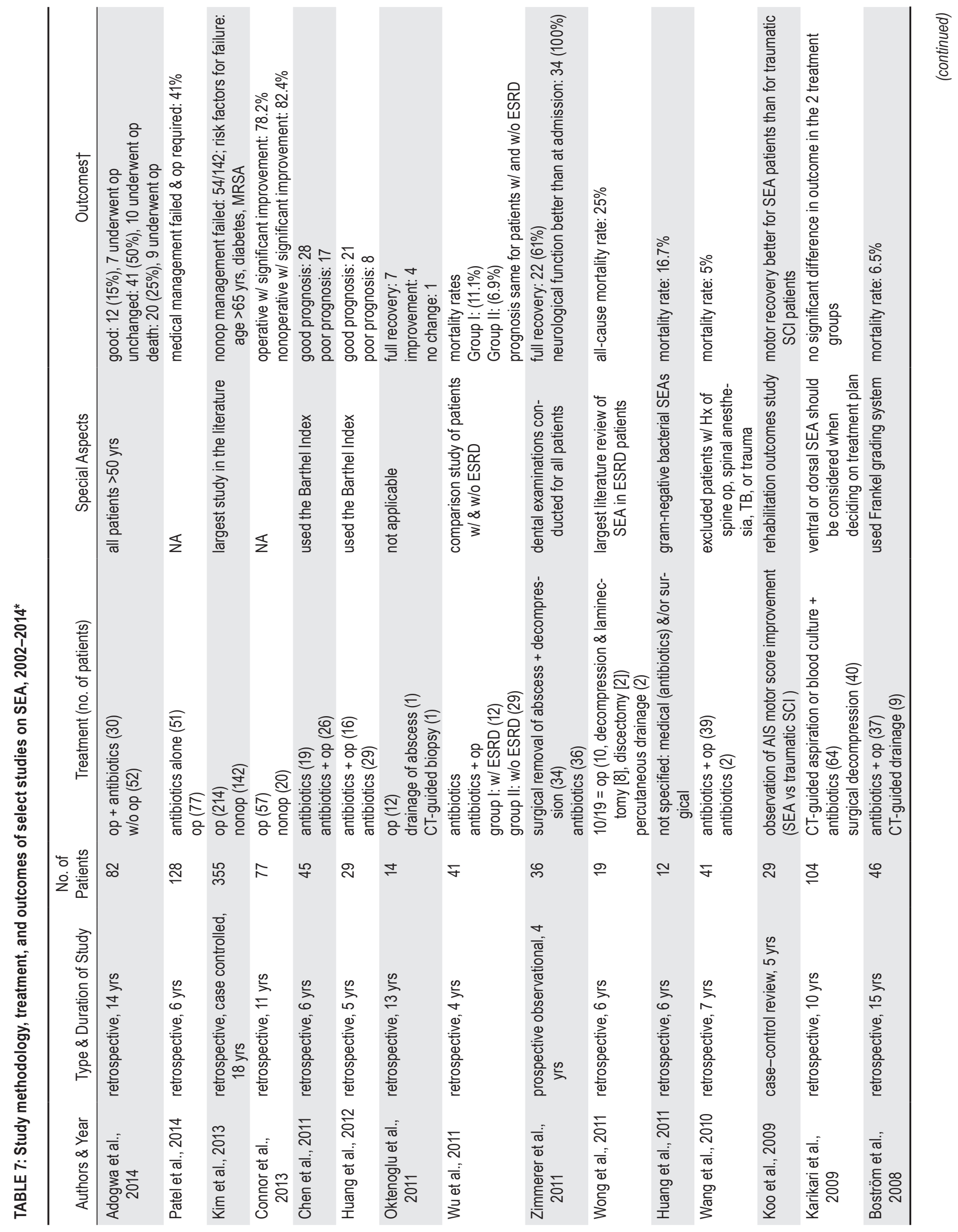


F. Shweikeh et al.

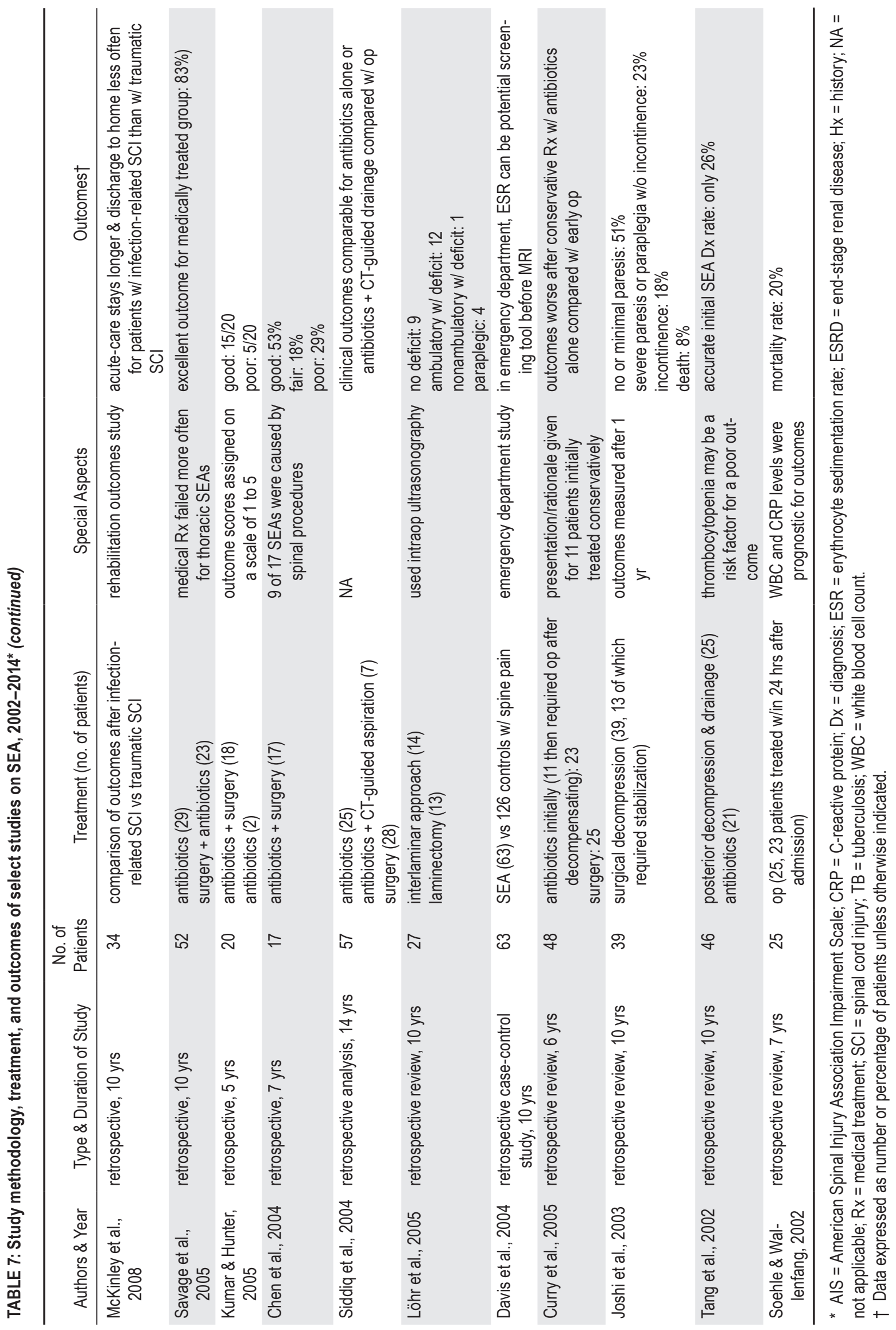


mography-guided percutaneous aspiration can be both diagnostic and therapeutic. ${ }^{9}$ In previous years, surgical intervention was the mainstay of management of this infection process, particularly when symptoms had not been present for longer than 36-72 hours., ${ }^{1,919,23,31,39}$ Later studies have shown successful outcomes with medical management alone. ${ }^{6,19,36,37}$ However, other more recent studies have indicated that early surgery has better outcomes. ${ }^{9,26,32,33}$ Others have demonstrated that medical treatment alone is more likely to fail among elderly patients or those with diabetes mellitus and/or MRSA infection..$^{23}$ The operative approach should be guided by the spatial orientation of the abscess. ${ }^{19}$ Instrumentation and fusion are usually avoided but might be required in rare cases..$^{19,31}$ Outcomes are mostly reported objectively, and obtaining long-term follow-up information for all patients can be difficult (Table 7). A few studies reporting long-term rehabilitative outcomes showed contradictory results. ${ }^{24,29}$

\section{Discussion}

It is widely acknowledged that diagnosis and treatment of SEAs should be conducted as soon as possible because of their potentially devastating neurological sequelae. Regarding diagnosis, the literature describes the most common presenting complaints as neck and back pain, but other reported signs and symptoms are neurological deficits, fever, altered mental status, and incontinence. ${ }^{4,10,19}$ Our study confirms all signs and symptoms described in the literature; back pain and neurological deficits (or both) were most common among patients in our study (experienced by almost half of patients).

Because of the nonspecific findings for SEA, a high index of suspicion is essential for patients with such symptoms. ${ }^{17,21}$ Such suspicion, along with early neuroimaging studies, is crucial for diagnosis and management. ${ }^{17}$ Certain predisposing conditions can further increase clinical suspicion. The literature cites the most common predisposing factor as being diabetes mellitus, along with previous spinal intervention, intravenous drug abuse, alcoholism, renal failure, bacteremia, and chronic inflammatory conditions. ${ }^{17,33}$ The patient data from our study parallel these findings. Epidural anesthesia has a wellestablished association with SEA (responsible for around $5 \%$ of all SEAs). ${ }^{3,5,21}$ A reported source of bacteremia and sepsis that can lead to development of SEAs is vascular access-related infection, notably in hemodialysis patients. $^{38,41}$ Clinicians should look for potential infectious origins such skin, oral, genitourinary, and/or gastrointestinal infections that can lead to bacteremia and a resulting SEA, as demonstrated by more than a third of the patients in our study.

Oktenoglu et al. reported that the most common location for SEAs was the thoracolumbar region. ${ }^{31}$ Our results build on this; in our study, the most common location for primary and secondary SEAs was the lumbar spine, followed by the thoracic spine. The mean number of vertebral levels spanned by the SEA was approximately 2 for both forms, with no significant difference. According to our literature search, Oktenoglu et al. were also the first to note that the location was dorsal in almost two-thirds of patients; they attributed this finding to hematogenous or contiguous spread from a paravertebral infection as well as the larger epidural space and venous plexus in this segment of the spine. ${ }^{2,31}$ Ventrally located SEAs are thought to be more rare and are usually associated with spondylitis or diskitis, although in that small retrospective study of 14 patients, the location was dorsal in only 6 patients. Patient data from our series revealed a prevalence of ventral SEAs (47 patients); SEAs were dorsal for only 30 patients (Table 2). These findings may be the result of a relatively high prevalence of osteomyelitis and diskitis in the studied population.

As has been found in other studies, $S$. aureus was the most common pathogen among patients in our study. A total of $75(70.0 \%)$ patients with SEAs had S. aureus infection, followed much further by Streptococcus spp. infection. Our data support the recommendation that antibiotic therapy including an antistaphylococcal agent should be initiated as soon as SEAs are diagnosed and then should be adjusted according to culture results. ${ }^{4,38}$ Although the period of antibiotic treatment is debatable, therapy should last longer than 4 weeks because therapy for less than 4 weeks is associated with a $25 \%$ rate of relapse. ${ }^{4,15,38}$ The neuroimaging study of choice is MRI because it provides the ability to visualize soft tissues as effectively as CT myelography with a comparable sensitivity for SEA (91\%-92\%); it offers the advantages of superior identification of perimedullary lesions and does not pose the risk of introducing pathogens into the thecal sac. ${ }^{41}$ MRI helps distinguish SEAs from other conditions in the differential diagnosis, which include tumor and subdural abscess. ${ }^{22} \mathrm{~A}$ characteristic description of an SEA on MR images includes a collection in the epidural space that is isointense or hyperintense on T1 images that enhances with gadolinium contrast and a T2 image with nonhomogeneous and hyperintense signal. ${ }^{33}$ A study by Tung et al. found that a poor outcome and/or lack of recovery to normal was associated with $50 \%$ narrowing of the spinal canal on initial MR images $(p=0.03)$, peripheral contrast enhancement $(\mathrm{p}=0.05)$, abnormal cord signal intensity, and an abscess longer than $3 \mathrm{~cm}(\mathrm{p}=0.01) .{ }^{41}$ In patients for whom MRI is contraindicated, other diagnostic modalities can be used, such as CT scanning, CTguided biopsy, and surgical exploration when none of the less invasive strategies are conclusive.

Surgical decompression has been suggested as the mainstay of therapy because symptoms result mainly from direct constriction of neural elements or the resultant ischemia. ${ }^{2,10,13,33,42}$ Most authors suggest that the upper time limit for consideration to intervene surgically is 3672 hours from onset of neurological sequelae. . $9,19,23,32,36,39$ Although for select patients a conservative approach of antibiotics alone might be appropriate,,$^{11,36,37}$ in our study, patients with focal neurological signs or symptoms were offered immediate surgical intervention. Those who had no response to antibiotics (as deemed by follow-up MRI) were also surgical candidates. Among those who received surgery along with antibiotics, 3.2\% died; among those who received antibiotics alone, $12.1 \%$ died, suggesting a potential mortality rate reduction with an aggressive surgical treatment approach. Nonetheless, such a finding 
needs to be further verified with future evidence through much larger studies.

In a larger study by Karikari et al., the operative approach was guided by the location of neural tissue compression. ${ }^{19}$ Dorsal SEAs were treated with posterior decompressive laminectomy with or without fusion. Ventral SEAs, particularly ventral cervical SEAs typically occurring in association with osteomyelitis or diskitis, were more likely to be treated by using an anterior approach because it allowed more thorough debridement and facilitated reconstruction of the anterior column. ${ }^{19,22,35}$ When an abscess was located dorsally, the treatment of choice was laminectomy, which has been identified as the most common procedure for thoracolumbar SEAs. ${ }^{22}$ These reported treatment approaches are similar to those used for the 106 patients in our study.

According to the literature, when dealing with an infected surgical site, spinal instrumentation is contraindicated because of increased risk for infection; however, spinal instrumentation might be necessary when extra support is needed for stabilization or when spinal stability is compromised from decompression..$^{31,35}$ In the Oktenoglu et al. study, ${ }^{31}$ metallic instrumentation was applied to 5 of 14 patients; the SEA was successfully treated for 4 of these patients. In our cohort, among the 19 patients who underwent fusion, the SEA was more likely to be ventral $(12[63.2 \%])$ and/or thoracic $(10[52.6 \%])$. Additionally, outcome was good for less than one-third of the patients (6 [31.6\%)]. Only 1 patient experienced SEA recurrence; however, obtaining long-term results with regard to reinfections and rehospitalizations in our patient population was difficult because many patients were lost to follow-up or received follow-up care at other institutions.

In our study, patient outcomes were based on longstanding neurological deficits and overall functional capacity. We reported outcomes objectively, in a manner similar to that described previously, ${ }^{25}$ which was suitable for the retrospective nature of our analysis. Of the patients not lost to follow-up, the frequency of good outcomes (full recovery, independent ambulation, no sphincter disturbance) was $37(60.7 \%)$; the rest of the patients $(39.3 \%)$ were determined to have poor outcomes (assisted ambulation, sphincter disturbance, paraplegia, bed confinement, or death).

\section{Literature Review}

Our goal for the literature review was to update current knowledge on nontuberculous bacterial SEAs. Particular attention was paid to the medical and surgical management of this condition, which is increasingly encountered by spine surgeons. Most of the studies reviewed were small, retrospective, single-center studies (Table 7). To the best of our knowledge, at the present time there is no consensus or guidelines for the treatment of SEAs. Clinical recommendations are based mostly on results of these studies and on expert opinions.

The most recent study reported results for elderly patients (> 50 years of age). ${ }^{1}$ Most $(>85 \%)$ of their cohort had either an unchanged condition or a poor outcome. The authors reported that for this age group, early surgical intervention lacked advantage compared with use of antibiotics alone. Although another very recent series showed that ideal treatment included surgery for those with focal deficits, age remains a factor; outcomes are better for younger patients. ${ }^{9}$ Yet another article recommends close attentiveness for those with nonoperative treatment because it is associated with a high rate of failure $(>40 \%)$. In the largest series reviewed (355 patients), Kim et al. showed that medical treatment is likely to fail for those older than 65 years of age and for those with MRSA infection, diabetes mellitus, and/or neurological symptoms..$^{23}$ On the basis of these findings, the authors provide a simple algorithm for determining the likelihood of failed medical management. The latter 2 studies reported high concordance between blood and abscess cultures, enabling clinicians to tailor antibiotics to blood culture results..$^{9,23}$

In 2011, Chen et al. conducted a 6-year retrospective review of 45 patients treated either with antibiotics alone or with antibiotics and surgery. ${ }^{8}$ They noted that the prognosis was worse for patients who at the time of initial presentation had diabetes mellitus, heart disease, higher age, and/or altered mental status. Outcomes were also poorer for those with late-stage disease, defined by the authors as neurological sequelae such as sensory impairment, weakness, bladder or bowel dysfunction, and/or paralysis. Huang et al. reported isolation of $S$. aureus from $29(69 \%)$ of 42 patients and gram-negative bacteria from $12(28.5 \%)$ of $42 .{ }^{16}$ This finding is consistent with findings previously reported in the literature and also with our finding that $S$. aureus is the most commonly isolated bacterium. Another study noted similar culprit microorganisms and reiterated that MRI should be performed for patients with back pain and fever, especially those with risk factors for a compromised immune system. ${ }^{17}$

$\mathrm{Wu}$ et al. compared outcomes for patients with and without end-stage renal disease. ${ }^{45}$ An attending neurosurgeon decided whether to treat with antibiotics alone or with antibiotics and surgical decompression. The prognosis remained unchanged for patients with and without endstage renal disease; however, in this patient population for whom infectious complications were a leading cause for illness and death, end-stage renal disease was a risk factor for repeat surgery. Wong et al. published similar results for a larger study in which mortality rate was higher among hemodialysis patients and likelihood for neurological improvement after medical or surgical intervention was decreased. ${ }^{44}$ In our study, $10(9.3 \%)$ SEA patients were receiving hemodialysis at the time of diagnosis. Of the 6 patients who died, 2 had received hemodialysis, adding to the many factors to be considered for SEA patients.

In a 4-year prospective observational study, of 36 patients, Zimmerer et al. described 34 who had undergone surgical SEA removal plus decompression followed by long-term antibiotic administration. ${ }^{46}$ This study was unique because all patients underwent clinical dental examinations with CT. Of the 36 patients, $22 \%$ had oral pathogenic bacteria. In 2010, Wang et al. ${ }^{43}$ conducted a 7-year retrospective study and discovered that poor motor recovery is correlated with poor glycemic control, prolonged muscle weakness before initiation of treatment, and poor response to treatment as measured by trends in 
white blood cell counts and C-reactive protein levels. In a larger study, Karikari et al. noted no significant difference in outcomes between 2 treatment groups: CT-guided aspiration plus antibiotics versus surgical decompression. ${ }^{19}$ They highlighted the importance of abscess location with regard to decision making. They found that patients with ventral SEA were more likely to show systemic signs and symptoms such as fever before neurological deficits and back pain (because of associated diskitis or osteomyelitis) and that patients with dorsal SEA were more likely to show neurological deficits. The former group can be treated conservatively with antibiotics and CT-guided aspiration, primarily because of early detection and significantly smaller SEAs.

In a series of 27 patients treated operatively, Löhr et al. ${ }^{26}$ demonstrated a strong advantage of surgical management. They advocated for an interlaminar decompressive approach, which provided more postoperative spinal stability and a similar likelihood of reoperation for recurrent or residual abscess when compared with laminectomy. In addition, they found that intraoperative ultrasonography enabled visualization of abscesses that might be hidden, thereby helping to reduce the likelihood of recurrence. A 10-year retrospective study noted excellent outcomes for $83 \%$ of medically treated patients. ${ }^{36}$ Selection criteria for the medically treated group included normal or stable neurological examination for more than 72 hours before admission (including signs and symptoms of radiculopathy or cord compression). Soehle and Wallenfang ${ }^{38}$ analyzed outcomes of 25 surgical patients after an average of 11 months. They found that poor outcomes were associated with higher preoperative and postoperative white blood cell counts (> 14,000 cells/ $\mu$ l), higher postoperative levels of C-reactive protein, a cervicothoracic location of the SEA, and lower limb deficits at the time of initial examination.

Studies have shown that misdiagnosis is common and can occur for up to $74 \%$ of patients, which leads to poorer outcomes. ${ }^{7,37}$ Chen et al. identified 3 of 17 patients with thoracic SEA who complained of abdominal symptoms, which led to misdiagnosis; they concluded that abdominal symptoms can mask neurological deficits and lead to delayed diagnosis. ${ }^{7}$ After a 10-year study, in 2003, Joshi et al. ${ }^{18}$ concluded that fever is not mandatory for the diagnosis of SEA. Because typically the first symptom of SEA is back pain, laboratory studies including erythrocyte sedimentation rate and C-reactive protein levels should be obtained. If those values are elevated, urgent MRI would help with early diagnosis of SEA. A later study builds on this finding, reporting that body temperature and white blood cell count within reference ranges do not preclude a diagnosis of SEA. ${ }^{10}$ Davis et al. ${ }^{12}$ reemphasize that erythrocyte sedimentation rate could be a potential screening tool before obtaining MRI for patients in the emergency room with suspected SEA.

Very few study reports comment on functional outcomes of SEA patients. Koo et al. ${ }^{24}$ studied neurological outcomes among patients with SEA-related myelopathy after an inpatient rehabilitation program and compared them with outcomes among patients with traumatic spinal cord injury. According to the American Spinal Injury
Association (ASIA) Impairment Scale scores, patients with SEA-related neurological deficits including complete motor or sensory loss showed significant improvement compared with patients with traumatic spinal cord injury. Overall, $73 \%$ of patients with SEA compared with only $9 \%$ of patients with traumatic spinal cord injury converted from complete ASIA Impairment Scale Grade A to incomplete ASIA Impairment Scale Grade B, C, or D. McKinley et al. ${ }^{29}$ compared outcomes of patients with traumatic spinal cord injury to outcomes of those with spinal infections; $85 \%$ of those patients with infections had SEAs. Patients with infections stayed in acute care significantly longer than did those with traumatic spinal cord injury; however, the length of stay in rehabilitation did not differ significantly between the 2 groups. In addition, among patients in the infections group, motor score improvements were lower and patients were less often discharged home than were those with traumatic spinal cord injury, reinforcing the importance of early patient and family education regarding potential future outcomes.

\section{Clinical Decision Making}

On the basis of our experience and on previous evidence from the literature, we present an algorithm for diagnosis and treatment of SEA (Fig. 3). Patients with suspicious signs and symptoms should undergo appropriate blood testing (e.g., white blood cell counts, erythrocyte sedimentation rates, C-reactive protein levels, cultures) and MRI; those with nonremarkable negative MRI findings should receive conservative treatment and monitoring. If imaging is suggestive of SEA, the decision for operative management should be based on the presence of factors that are highly associated with failure of medical treatment alone (i.e., diabetes mellitus, age $>65$ years, neurological deficits [for < 36-72 hours], and MRSA-positive blood culture results). Otherwise, medical treatment with antibiotics only can be confidently started. These patients should undergo CT-guided biopsy of the lesion, especially if blood cultures are inconclusive. They also need to be monitored for signs of medical treatment failure, such as onset of neurological compromise and persistence of elevated inflammatory markers (C-reactive protein levels, white blood cell count), blood culture positivity, and imaging indications of SEA. If signs of medical failure are detected, surgical decompression should be initiated promptly.

\section{Limitations}

A limitation encountered in our analysis and in published SEA studies was lack of complete long-term follow-up data. After discharge, a number of patients were transferred to other facilities. These limitations impede the ability to obtain coherent assumptions on outcomes of each treatment modality. In addition, the retrospective nature of these studies further limits their interpretation. Errors in the data and in data collection can and do occur. As best we can determine, advances in imaging modalities and treatment strategies have decreased deaths from this serious infection. As such enhancements continue to expand, future multicenter studies with complete longterm follow-up can augment our understanding of the condition. 


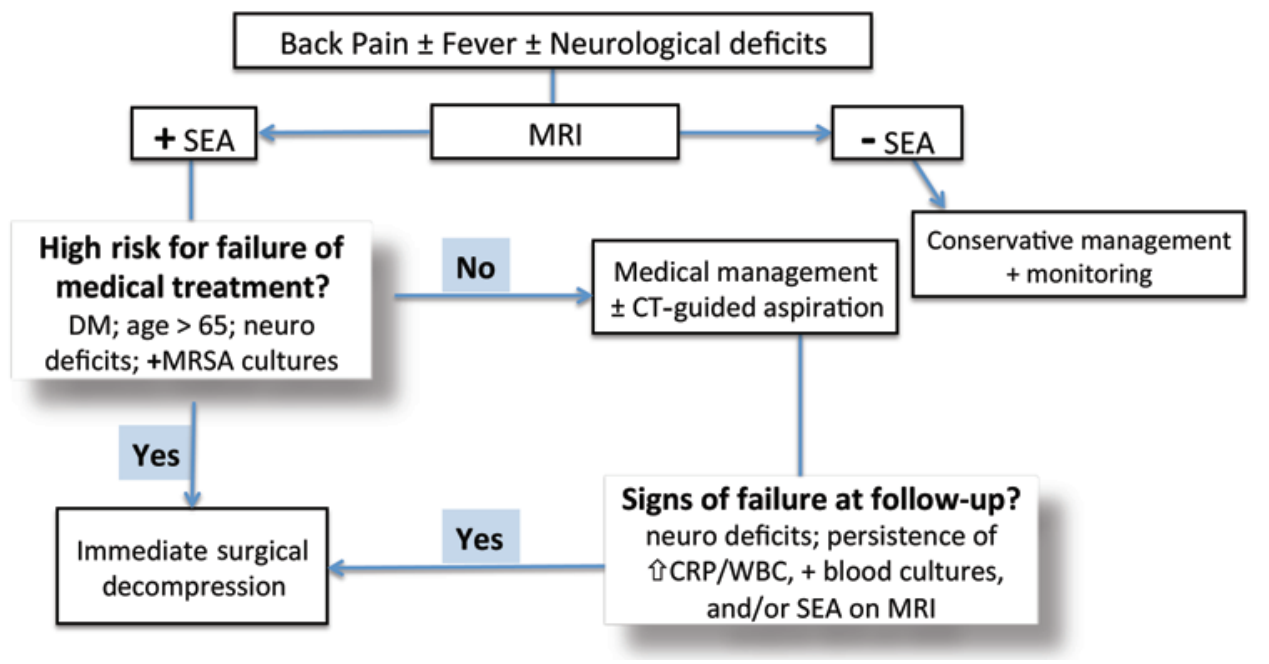

FIG. 3. Algorithm for diagnosis and treatment of SEA. CRP = C-reactive protein levels; $D M=$ diabetes mellitus; neuro = neurological; WBC = white blood cell count.

\section{Conclusions}

Prompt diagnosis and treatment of bacterial SEA can improve outcomes, but the outcome will be poor for a substantial percentage of patients. Those with multiple risk factors-older age, diabetes mellitus, end-stage renal disease, and intravenous drug abuse-are predisposed to this rare infection and to having worse outcomes. The organism most frequently responsible for SEA is $S$. aureus and MRSA has been correlated with a higher rate of treatment failure. Optimum care involves diagnosis with MRI, administration of appropriate antibiotics with or without surgery, and appreciation of the effects of concurrent conditions and risk factors. After the decision to operate has been made, surgery should be performed immediately and no longer than 36-72 hours after onset of neurological symptoms. Although management with surgical decompression was associated with lower mortality rates in our study and better outcomes in studies reported in the literature, multiple studies also support use of antibiotic therapy alone. Undoubtedly, neurological status at the time of presentation is a key factor in decision making and patient outcome. Overall, management decisions entail individual patient considerations. Although this aspect of the condition is a challenge for spine surgeons, an evidence-based algorithm is proposed. We hope that it will be a valuable tool for clinicians. Future large studies with long-term follow-up can verify the utility of this algorithm and can establish a consensus-based protocol for this increasingly prevalent condition.

\section{Acknowledgment}

We thank Dr. Adrian Ochoa for assistance with data collection and Dr. David Sperling for his support with the institutional review board.

\section{Disclosure}

The authors report no conflict of interest concerning the materials or methods used in this study or the findings specified in this paper.
Author contributions to the study and manuscript preparation include the following. Conception and design: Shweikeh. Acquisition of data: Shweikeh, Saeed, Bukavina, Zyck. Analysis and interpretation of data: Shweikeh, Saeed, Zyck. Drafting the article: Shweikeh, Saeed, Zyck. Critically revising the article: Shweikeh, Bukavina, Drazin, Steinmetz. Reviewed submitted version of manuscript: Shweikeh, Drazin, Steinmetz. Approved the final version of the manuscript on behalf of all authors: Shweikeh. Statistical analysis: Bukavina. Study supervision: Shweikeh, Steinmetz.

\section{References}

1. Adogwa O, Karikari IO, Carr KR, Krucoff M, Ajay D, Fatemi $\mathrm{P}$, et al: Spontaneous spinal epidural abscess in patients 50 years of age and older: a 15-year institutional perspective and review of the literature. Clinical article. J Neurosurg Spine 20:344-349, 2014

2. Batson OV: The vertebral vein system. Caldwell lecture, 1956. Am J Roentgenol Radium Ther Nucl Med 78:195-212, 1957

3. Beaudoin MG, Klein L: Epidural abscess following multiple spinal anaesthetics. Anaesth Intensive Care 12:163-164, 1984

4. Boström A, Oertel M, Ryang Y, Rohde V, Bürgel U, Krings T, et al: Treatment strategies and outcome in patients with nontuberculous spinal epidural abscess-a review of 46 cases. Minim Invasive Neurosurg 51:36-42, 2008

5. Burstal R, Wegener F, Hayes C, Lantry G: Epidural analgesia: prospective audit of 1062 patients. Anaesth Intensive Care 26:165-172, 1998

6. Butler JS, Shelly MJ, Timlin M, Powderly WG, O’Byrne JM: Nontuberculous pyogenic spinal infection in adults: a 12-year experience from a tertiary referral center. Spine (Phila Pa 1976) 31:2695-2700, 2006

7. Chen HC, Tzaan WC, Lui TN: Spinal epidural abscesses: a retrospective analysis of clinical manifestations, sources of infection, and outcomes. Chang Gung Med J 27:351-358, 2004

8. Chen SH, Chang WN, Lu CH, Chuang YC, Lui CC, Chen SF, et al: The clinical characteristics, therapeutic outcome, and prognostic factors of non-tuberculous bacterial spinal epidural abscess in adults: a hospital-based study. Acta Neurol Taiwan 20:107-113, 2011

9. Connor DE Jr, Chittiboina P, Caldito G, Nanda A: Comparison of operative and nonoperative management of spinal epidural abscess: a retrospective review of clinical and laboratory predictors of neurological outcome. Clinical article. J Neurosurg Spine 19:119-127, 2013

10. Curry WT Jr, Hoh BL, Amin-Hanjani S, Eskandar EN: Spinal 
epidural abscess: clinical presentation, management, and outcome. Surg Neurol 63:364-371, 2005

11. Darouiche RO: Spinal epidural abscess. N Engl J Med 355: 2012-2020, 2006

12. Davis DP, Wold RM, Patel RJ, Tran AJ, Tokhi RN, Chan TC, et al: The clinical presentation and impact of diagnostic delays on emergency department patients with spinal epidural abscess. J Emerg Med 26:285-291, 2004

13. Du Pen SL, Peterson DG, Williams A, Bogosian AJ: Infection during chronic epidural catheterization: diagnosis and treatment. Anesthesiology 73:905-909, 1990

14. González-López JJ, Górgolas M, Muñiz J, López-Medrano F, Barnés PR, Fernández Guerrero ML: Spontaneous epidural abscess: analysis of 15 cases with emphasis on diagnostic and prognostic factors. Eur J Intern Med 20:514-517, 2009

15. Hadjipavlou AG, Mader JT, Necessary JT, Muffoletto AJ: Hematogenous pyogenic spinal infections and their surgical management. Spine (Phila Pa 1976) 25:1668-1679, 2000

16. Huang CR, Lu CH, Chuang YC, Chen SF, Tsai NW, Chang $\mathrm{CC}$, et al: Clinical characteristics and therapeutic outcome of Gram-negative bacterial spinal epidural abscess in adults. J Clin Neurosci 18:213-217, 2011

17. Huang PY, Chen SF, Chang WN, Lu CH, Chuang YC, Tsai NW, et al: Spinal epidural abscess in adults caused by Staphylococcus aureus: clinical characteristics and prognostic factors. Clin Neurol Neurosurg 114:572-576, 2012

18. Joshi SM, Hatfield RH, Martin J, Taylor W: Spinal epidural abscess: a diagnostic challenge. Br J Neurosurg 17:160-163, 2003

19. Karikari IO, Powers CJ, Reynolds RM, Mehta AI, Isaacs RE: Management of a spontaneous spinal epidural abscess: a singlecenter 10-year experience. Neurosurgery 65:919-924, 2009

20. Khan SH, Hussain MS, Griebel RW, Hattingh S: Title comparison of primary and secondary spinal epidural abscesses: a retrospective analysis of 29 cases. Surg Neurol 59:28-33, 2003

21. Khanna RK, Malik GM, Rock JP, Rosenblum ML: Spinal epidural abscess: evaluation of factors influencing outcome. Neurosurgery 39:958-964, 1996

22. Kim CW, Currier BL, Heller JG, Eismont FJ: Cervical spine infections, in Benzel EC, Connolly PJ, DiAngelo DJ, et al (eds): The Cervical Spine, ed 5. Philadelphia: Lippincott Williams \& Wilkins, 2012, pp 782-808

23. Kim SD, Melikian R, Ju KL, Zurakowski D, Wood KB, Bono $\mathrm{CM}$, et al: Independent predictors of failure of nonoperative management of spinal epidural abscesses. Spine $\mathbf{J}$ [epub ahead of print], 2013

24. Koo DW, Townson AF, Dvorak MF, Fisher CG: Spinal epidural abscess: a 5-year case-controlled review of neurologic outcomes after rehabilitation. Arch Phys Med Rehabil 90:512516,2009

25. Kumar K, Hunter G: Spinal epidural abscess. Neurocrit Care 2:245-251, 2005

26. Löhr M, Reithmeier T, Ernestus RI, Ebel H, Klug N: Spinal epidural abscess: prognostic factors and comparison of different surgical treatment strategies. Acta Neurochir (Wien) 147:159-166, 2005

27. Lu CH, Chang WN, Lui CC, Lee PY, Chang HW: Adult spinal epidural abscess: clinical features and prognostic factors. Clin Neurol Neurosurg 104:306-310, 2002

28. Mackenzie AR, Laing RB, Smith CC, Kaar GF, Smith FW: Spinal epidural abscess: the importance of early diagnosis and treatment. J Neurol Neurosurg Psychiatry 65:209-212, 1998

29. McKinley W, Merrell C, Meade M, Brooke K, DiNicola A: Rehabilitation outcomes after infection-related spinal cord disease: a retrospective analysis. Am J Phys Med Rehabil 87: 275-280, 2008
30. Miftode E, Luca V, Mihalache D, Leca D, Stefanidis E, Anuţa C, et al: Spinal epidural abscess. Rev Med Chir Soc Med Nat Iasi 105:778-784, 2001

31. Oktenoglu T, Sasani M, Cetin B, Bozkus H, Ercelen O, Vural $\mathrm{M}$, et al: Spontaneous pyogenic spinal epidural abscess. Turk Neurosurg 21:74-82, 2011

32. Patel AR, Alton TB, Bransford RJ, Lee MJ, Bellabarba CB, Chapman JR: Spinal epidural abscesses: risk factors, medical versus surgical management, a retrospective review of 128 cases. Spine J 14:326-330, 2014

33. Pereira CE, Lynch JC: Spinal epidural abscess: an analysis of 24 cases. Surg Neurol 63 (Suppl 1):S26-S29, 2005

34. Reihsaus E, Waldbaur H, Seeling W: Spinal epidural abscess: a meta-analysis of 915 patients. Neurosurg Rev 23:175-205, 2000

35. Rhee JM, Heller JG: Spinal infections, in Frymoyer JW, Wiesel SW, An HS, et al (eds): The Adult and Pediatric Spine: An Atlas of Differential Diagnosis, ed 3. Philadelphia: Lippincott Williams \& Wilkins, 2004, Vol 1, pp 165-190

36. Savage K, Holtom PD, Zalavras CG: Spinal epidural abscess: early clinical outcome in patients treated medically. Clin Orthop Relat Res 439:56-60, 2005

37. Siddiq F, Chowfin A, Tight R, Sahmoun AE, Smego RA Jr: Medical vs surgical management of spinal epidural abscess. Arch Intern Med 164:2409-2412, 2004

38. Soehle M, Wallenfang T: Spinal epidural abscesses: clinical manifestations, prognostic factors, and outcomes. Neurosurgery 51:79-87, 2002

39. Sørensen P: Spinal epidural abscesses: conservative treatment for selected subgroups of patients. Br J Neurosurg 17:513518,2003

40. Tang HJ, Lin HJ, Liu YC, Li CM: Spinal epidural abscessexperience with 46 patients and evaluation of prognostic factors. J Infect 45:76-81, 2002

41. Tung GA, Yim JW, Mermel LA, Philip L, Rogg JM: Spinal epidural abscess: correlation between MRI findings and outcome. Neuroradiology 41:904-909, 1999

42. Turgut M: Complete recovery of acute paraplegia due to pyogenic thoracic spondylodiscitis with an epidural abscess. Acta Neurochir (Wien) 150:381-386, 2008

43. Wang TC, Lu MS, Yang JT, Weng HH, Cheng YK, Lin MH, et al: Motor function improvement in patients undergoing surgery for spinal epidural abscess. Neurosurgery 66:910-916, 2010

44. Wong SS, Daka S, Pastewski A, Kyaw W, Chapnick E, Sepkowitz D: Spinal epidural abscess in hemodialysis patients: a case series and review. Clin J Am Soc Nephrol 6:1495-1500, 2011

45. Wu MY, Fu TS, Chang CH, Hsu HH, Chang MY, Tian YC, et al: Aggressive surgical intervention in end-stage renal disease patients with spinal epidural abscess. Ren Fail 33:582-586, 2011

46. Zimmerer SM, Conen A, Müller AA, Sailer M, Taub E, Flückiger U, et al: Spinal epidural abscess: aetiology, predisponent factors and clinical outcomes in a 4-year prospective study. Eur Spine J 20:2228-2234, 2011

Manuscript submitted April 16, 2014.

Accepted June 10, 2014.

Please include this information when citing this paper: DOI: 10.3171/2014.6.FOCUS14146.

Address correspondence to: Faris Shweikeh, M.D., College of Medicine, Northeast Ohio Medical University, 4209 State Rte. 44, Rootstown, OH 44272. email: faris.shweikeh@gmail.com. 